\title{
Shape Correspondence through Landmark Sliding
}

\author{
Song Wang, Toshiro Kubota, and Theodor Richardson \\ Department of Computer Science and Engineering \\ University of South Carolina, Columbia, SC 29208, U.S.A. \\ songwang@cse.sc.edu, kubota@cse.sc.edu, richa268@cse.sc.edu
}

\begin{abstract}
Motivated by improving statistical shape analysis, this paper presents a novel landmark-based method for accurate shape correspondence, where the general goal is to align multiple shape instances by corresponding a set of given landmark points along those shapes. Different from previous methods, we consider both global shape deformation and local geometric features in defining the shapecorrespondence cost function to achieve a consistency between the landmark correspondence and the underlying shape correspondence. According to this cost function, we develop a novel landmark-sliding algorithm to achieve optimal landmark-based shape correspondence with preserved shape topology. The proposed method can be applied to correspond various $2 D$ shapes in the forms of single closed curves, single open curves, self-crossing curves, and multiple curves. We also discuss the practical issue of landmark initialization. The proposed method has been tested on various biological shapes arising from medical image analysis and validated in constructing statistical shape models.
\end{abstract}

\section{Introduction}

Geometric shape information plays a key role in many computer vision and image processing applications, especially in medical image analysis where many anatomic structures and related functions can be identified and classified in terms of their unique shapes. In many applications, we need to analyze the shape of the same structure or object across a group of individuals to construct a deformable or dynamic shape model. This leads to the development of many useful methods on statistical shape analysis $[2,5,14,9,11]$. However, most, if not all, of those methods require the shape instances to be first accurately aligned in the form of some sparsely-sampled landmarks, or landmark-based shape correspondence.

At the foundation of shape correspondence is a definition of the shape difference that measures the nonrigid deformation or variation from one shape to another. Shape difference is usually conveniently defined in terms of landmarkbased shape representation. In this case, shape correspondence can be defined as identifying a set of landmarks along shapes such that: (a) those landmarks well represent the original shapes, and (b) the total shape difference (based on landmarks) across those shapes is minimized. As in statistical shape analysis, landmarks as discussed in this paper refer to a set of sampling points along the shape contours and may not coincide with anatomically critical points.

A large group of landmark-based shape correspondence methods $[7,4,1,12]$ can be categorized as "shape matching”, where all the landmark points are pre-sampled and fixed during the correspondence. Various shape matching algorithms only find matched landmarks from one shape to another. In general, landmark-based shape matching methods can be divided into two groups in terms of the features used in defining shape difference. The first group of methods [7, 12] locates the matching landmarks using local geometric features like curve curvature, arc-length, curve smoothness, and local convexity. To preserve the shape topology, dynamic programming techniques are usually adopted to achieve an optimal correspondence. Localfeature shape matching is not effective to capture the nonrigid global shape variation that is common in biological and medical shape analysis. The second group of methods $[4,1]$ propose some global shape-difference measures to match landmarks. Without locality in the cost function, global shape matching usually results in some nonlinear optimization problem, which is prone to be trapped in some local optima. Both local and global shape matching methods suffer from the problem of correspondence accuracy because the landmarks are pre-sampled and fixed. As a result, shape matching methods are mainly used in applications like shape recognition or shape-based retrieval, where quantitatively accurate shape description is not required.

Accurate landmark-based shape correspondence [13, 3 , $10,8,15,6]$ attracts extensive attention following the progress and popularity of statistical shape analysis [5, 2, 9]. Many researchers $[6,3]$ pointed out that the accuracy of shape correspondence greatly affects the accuracy and reliability of statistical shape modelling. Small error in shape correspondence may enormously degrade the resulting shape model. A series of landmark-based shapecorrespondence methods $[10,8,6]$ have been developed as an important component in point distribution models [5], where the variance of all the landmarks is directly used to measure the shape-correspondence error. Those methods 
construct a very complicated cost function that can only be locally optimized using random searching algorithms, such as genetic algorithms and simulated annealing methods.

Most closely related to our research is the splinerelaxation method proposed by Bookstein [3], where initial landmarks are allowed to slide along the estimated tangent directions to reduce the shape-difference measure. Similar to our approach, this spline-relaxation method also adopts the thin-plate spline for modelling the shape difference. However, this relaxation method moves landmarks away from the underlying shape contour and, therefore, the resulting landmarks may not represent the original shape contours very well. Another method closely related to our approach is the curve-mapping method developed by Powell [13], where thin-plate splines are also used for measuring shape difference and a nonlinear optimization software package is used to find a local optimal correspondence. But this curvemapping method does not consider whether the resulting landmarks are well distributed along the shape contours to represent the original shapes.

In this paper, we develop a new landmark-sliding method for shape correspondence. One main contribution of this paper is the definition of a new shape-correspondence cost function that combines the global features and local features underlying the landmark-based shapes. While the global features catch nonrigid deformation between shapes, the local features constrain the distribution of the sampled landmarks along the shape contours to keep the consistency between landmark correspondence and the desired shape correspondence. Based on this cost function, we develop an iterative algorithm, which slides the landmarks along the shape contours to achieve the landmark correspondence. With an additional projection step, this method avoids moving landmarks away from the underlying shape contour.

The rest of the paper is organized as follows. In Section 2, we describe the proposed shape-correspondence cost function and landmark-sliding method for two single closed-curve shapes. Section 3 generalizes the proposed method to more complex shapes, presents representative results in medical image analysis, and discusses some related issues in practical applications. A brief conclusion is given in Section 4.

\section{The Proposed Method}

\subsection{Problem formulation}

In this section we develop and describe a method to correspond a target shape onto a template shape, both of which, for simplicity, are made of a closed curve with known parametric representation. Furthermore, landmarks in the template shape are given and our task is to identify a set of corresponding landmarks in the target shape from a set of initial landmarks. Later in Section 3, we will further discuss the extension of the proposed method to more general cases like open-curve shapes, self-crossing shapes, multiple-curve shapes, and multiple-shape correspondence. Various shape examples are illustrated in Fig. 1.

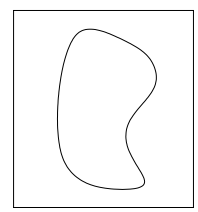

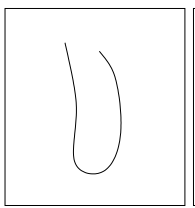

(b)

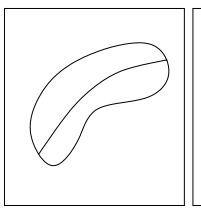

(c)

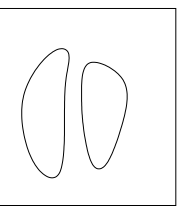

Figure 1: Illustrations of different shapes: (a) single closedcurve shape, (b) single open-curve shape, (c) self-crossing shape, and (d) multiple-curve shape.

Denote a parametric representation of the template shape $\mathbf{u}(t)=(\hat{x}(t), \hat{y}(t))^{T}, 0 \leq t \leq \hat{L}$ and the target shape $\mathbf{v}(s)=(x(s), y(s))^{T}, 0 \leq s \leq L$, where $\hat{L}$ and $L$ are the total curve lengths of the template and the target shapes, respectively. With the assumption of closed shapes, we have $\mathbf{u}(0)=\mathbf{u}(\hat{L})$ and $\mathbf{v}(0)=\mathbf{v}(L)$. In this parametrization, $t \mid \hat{L}$ represents the traversed curve length from $\mathbf{u}(0)$ to $\mathbf{u}(t)$ and $s \mid L$ represents the traversed curve length from $\mathbf{v}(0)$ to $\mathbf{v}(s)$, where $a \mid b$ is the modulus operation.

Let $\mathbf{t}=\left\{t_{0}, t_{1}, \ldots, t_{n-1}\right\}$ be a set of parameters which generates $n$ sequentially-sampled landmarks $\mathbf{U}=\left\{\mathbf{u}\left(t_{0}\right), \mathbf{u}\left(t_{1}\right), \ldots, \mathbf{u}\left(t_{n-1}\right)\right\}$. We assume that those landmarks represent the template shape well. Shapecorrespondence is to seek in the target shape $n$ parameters $\mathbf{s}=\left\{s_{0}, s_{1}, \ldots, s_{n-1}\right\}$ such that the landmarks $\mathbf{V}=$ $\left\{\mathbf{v}\left(s_{0}\right), \mathbf{v}\left(s_{1}\right), \ldots, \mathbf{v}\left(s_{n-1}\right)\right\}$ match the landmarks $\mathbf{U}$ in the template shape. According to the statistical shape theory [9], $\mathbf{U}$ and $\mathbf{V}$ can be regarded as the landmark representation of the template and the target shapes, respectively. Obviously, an important requirement is that the identified $\mathbf{V}$ also represents well the underlying target shape $\mathbf{v}(s), 0 \leq s \leq L$.

In this paper, shape correspondence is formulated as seeking the optimal parameters $\mathbf{s}$ to minimize the cost function

$$
\phi(\mathbf{s})=d(\mathbf{U}, \mathbf{V})+\lambda R(\mathbf{s})
$$

subject to some additional constraints to preserve the shape topology, i.e., landmarks $\mathbf{v}\left(s_{0}\right), \mathbf{v}\left(s_{1}\right), \ldots, \mathbf{v}\left(s_{n-1}\right)$ should be sequentially located along the target shape contour as $\mathbf{U}$ along the template shape. In this formulation, $d(\mathbf{U}, \mathbf{V})$ is called the (landmark-based) shape difference which measures the global shape deformation between the template and target shapes. $R(\mathbf{s})$ is the representation error in using $n$ landmarks $\mathbf{V}$ to represent the underlying target shape. We desire small representation error $R(\mathbf{s})$ such that landmarkbased shape difference $d(\mathbf{U}, \mathbf{V})$ reflects the underlying shape correspondence error.

This formulation brings us several key issues which need to be addressed: (a) definition of the landmark-based shape 
difference $d(\mathbf{U}, \mathbf{V})$, (b) selection of landmark representation error $R(\mathbf{s})$, (c) introduction of an additional constraint to preserve the shape topology, and (d) development of effective algorithms to find the optima of the shapecorrespondence cost function.

\subsection{Shape difference measure $d(\mathbf{U}, \mathbf{V})$}

For brevity, let's denote $\mathbf{u}\left(t_{i}\right)$ as $\mathbf{u}_{i}=\left(\hat{x}_{i}, \hat{y}_{i}\right)^{T}$ and $\mathbf{v}\left(s_{i}\right)$ as $\mathbf{v}_{i}=\left(x_{i}, y_{i}\right)^{T}, i=0,1, \ldots, n-1$. The shape difference between $\mathbf{U}$ and $\mathbf{V}$ is measured by deforming $\mathbf{V}$ to $\mathbf{U}$ using a 2-D thin-plate spline model [2]. This deformation is characterized by $\mathbf{h}=(f, g)^{T}: \mathbb{R}^{2} \rightarrow \mathbb{R}^{2}$ such that $\mathbf{V}=\mathbf{h}(\mathbf{U})$, i.e., $\mathbf{v}_{i}=\mathbf{h}\left(\mathbf{u}_{i}\right), i=0,1, \ldots, n-1$, where

$$
\left\{\begin{array}{l}
f(\mathbf{u})=a_{0}+a_{1} \hat{x}+a_{2} \hat{y}+\sum_{i=0}^{n-1} c_{i} K\left(\mathbf{u}, \mathbf{u}_{i}\right) \\
g(\mathbf{u})=b_{0}+b_{1} \hat{x}+b_{2} \hat{y}+\sum_{i=0}^{n-1} d_{i} K\left(\mathbf{u}, \mathbf{u}_{i}\right) .
\end{array}\right.
$$

The parameters $\mathbf{a}=\left(a_{0}, a_{1}, a_{2}\right)^{T}, \mathbf{b}=\left(b_{0}, b_{1}, b_{2}\right)^{T}$, $\mathbf{c}=\left(c_{0}, c_{1}, \ldots, c_{n-1}\right)^{T}$, and $\mathbf{d}=\left(d_{0}, d_{1}, \ldots, d_{n-1}\right)^{T}$ in Eq. (1) can be calculated by solving the following matrix equation:

$$
\left(\begin{array}{cc}
\mathbf{K} & \mathbf{P} \\
\mathbf{P}^{T} & \mathbf{0}
\end{array}\right)\left(\begin{array}{ll}
\mathbf{c} & \mathbf{d} \\
\mathbf{a} & \mathbf{b}
\end{array}\right)=\left(\begin{array}{cc}
\mathbf{x} & \mathbf{y} \\
\mathbf{0} & \mathbf{0}
\end{array}\right),
$$

where $k_{i j}=K\left(\mathbf{u}_{i}, \mathbf{u}_{j}\right)=\left\|\mathbf{u}_{i}-\mathbf{u}_{j}\right\|^{2} \log \left\|\mathbf{u}_{i}-\mathbf{u}_{j}\right\|$, $i, j=0,1, \ldots, n-1$, and $\mathbf{P}=(\mathbf{1}, \hat{\mathbf{x}}, \hat{\mathbf{y}})$. Note that $\mathbf{x}=\left(x_{0}, x_{1}, \ldots, x_{n-1}\right)^{T}, \mathbf{y}=\left(y_{0}, y_{1}, \ldots, y_{n-1}\right)^{T}, \hat{\mathbf{x}}=$ $\left(\hat{x}_{0}, \hat{x}_{1}, \ldots, \hat{x}_{n-1}\right)^{T}$, and $\hat{\mathbf{y}}=\left(\hat{y}_{0}, \hat{y}_{1}, \ldots, \hat{y}_{n-1}\right)^{T}$, where $\mathbf{u}_{i}=\left(\hat{x}_{i}, \hat{y}_{i}\right)$ and $\mathbf{v}_{i}=\left(x_{i}, y_{i}\right), i=0,1, \ldots, n-1$ are corresponding landmarks between $\mathbf{U}$ and $\mathbf{V}$. It can be shown that the above transform minimizes the following so-called bending energy function [2]

$$
\phi(\mathbf{h})=\iint_{-\infty}^{\infty}(L(f)+L(g)) d x d y
$$

where $L(\cdot)=\left(\frac{\partial^{2}}{\partial x^{2}}\right)^{2}+2\left(\frac{\partial^{2}}{\partial x \partial y}\right)^{2}+\left(\frac{\partial^{2}}{\partial y^{2}}\right)^{2}$.

Substituting (1) and (2) into (3) yields

$$
\phi(\mathbf{h})=\mathbf{c}^{T} \mathbf{K} \mathbf{c}+\mathbf{d}^{T} \mathbf{K} \mathbf{d}=\frac{1}{8 \pi}\left(\mathbf{x}^{T} \mathbf{L} \mathbf{x}+\mathbf{y}^{T} \mathbf{L} \mathbf{y}\right)
$$

where $\mathbf{L}$ is the $n \times n$ upper left submatrix of

$$
\left(\begin{array}{cc}
\mathbf{K} & \mathbf{P} \\
\mathbf{P}^{T} & \mathbf{0}
\end{array}\right)^{-1}
$$

Here $\mathbf{L}$ is positive semidefinite because the thin-plate bending energy is invariant to affine transforms. This makes the thin-plate splines especially suitable for describing nonrigid shape deformations in biological and medical applications. We can directly use the bending energy as $d(\mathbf{U}, \mathbf{V})$, i.e.,

$$
d(\mathbf{U}, \mathbf{V})=\frac{1}{8 \pi}\left\{\mathbf{x}^{T} \mathbf{L} \mathbf{x}+\mathbf{y}^{T} \mathbf{L y}\right\}
$$

\subsection{Shape representation error $R(\mathbf{s})$}

Let $l_{i}, i=0,1, \ldots n-1$ be the traversed curve length from $\mathbf{v}_{i}$ to $\mathbf{v}_{i+1 \mid n}$. It is easy to see that

$$
l_{i}=\left(s_{i+1 \mid n}-s_{i}\right) \mid L .
$$

Similarly, we have the corresponding curve length between neighboring landmarks in the template shape

$$
\hat{l}_{i}=\left(t_{i+1 \mid n}-t_{i}\right) \mid \hat{L}
$$

Obviously, we have

$$
0<l_{i}<L, 0<\hat{l}_{i}<\hat{L}, i=0,1, \ldots, n-1 \text {. }
$$

With the assumption that $\mathbf{u}_{0}, \mathbf{u}_{1}, \ldots, \mathbf{u}_{n-1}$ are consecutively sampled landmarks along the closed template shape contour, we know that

$$
\sum_{i=0}^{n-1} \hat{l}_{i}=\hat{L}
$$

In order to improve the representation accuracy, this paper desires a similar spatial landmark distribution along the template and target shapes. Specifically, we expect

$$
\frac{l_{i}}{l_{i+1 \mid n}} \approx \frac{\hat{l}_{i}}{\hat{l}_{i+1 \mid n}}, i=0,1, \ldots, n-1 \text {. }
$$

Following this principle, this paper defines the landmark representation error in the target shape as

$$
R(\mathbf{s})=\sum_{i=0}^{n-1}\left(l_{i} \hat{l}_{i+1 \mid n}-l_{i+1 \mid n} \hat{l}_{i}\right)^{2}
$$

Unlike the shape difference measure $d(\mathbf{U}, \mathbf{V})$, this representation error only involves with local geometric features.

\subsection{Topology-preserving constraint}

As mentioned before, we require that $\mathbf{v}_{0}, \mathbf{v}_{1}, \ldots, \mathbf{v}_{n-1}$ are also consecutively distributed in the target shape as those in the template shape. Otherwise, the shape topology of the template and target would be different from each other. Similar to (5), we impose the following constraint to the shape correspondence cost function.

$$
\sum_{i=0}^{n-1} l_{i}=L .
$$

This means every point along the target shape contour is traversed only once when we start from $\mathbf{v}_{0}$, sequentially pass through all landmarks in $\mathbf{V}$, and finally get back to $\mathbf{v}_{0}$. 
Combining the above selections of shape-difference measure, shape-representation error, and the additional constraints, the landmark-based shape-correspondence problem can be described as

$$
\begin{aligned}
\min _{\mathbf{s}} & \left\{\left(\mathbf{x}^{T} \mathbf{L} \mathbf{x}+\mathbf{y}^{T} \mathbf{L} \mathbf{y}\right)\right. \\
& \left.+\lambda \sum_{i=0}^{n-1}\left(l_{i} \hat{l}_{i+1 \mid n}-l_{i+1 \mid n} \hat{l}_{i}\right)^{2}\right\}
\end{aligned}
$$

subject to

$$
\begin{aligned}
& 0<s_{i}<\hat{L} \\
& l_{i}=\left(s_{i+1 \mid n}-s_{i}\right) \mid L, i=0,1, \ldots, n-1 \\
& \sum_{i=0}^{n-1} l_{i}=L
\end{aligned}
$$

where $\lambda>0$ is a regularization factor which balances the contribution from the shape difference and the shape representation. The following section is focused on developing algorithms to solve this optimization problem. Note that both the current problem formulation and the following algorithm are for the cases of single closed-curve shapes. Generalization to more general cases will be discussed in Section 3.

\subsection{Landmark sliding algorithm}

The landmark-based shape-correspondence problem formulated above is a constrained nonlinear optimization problem because the parametric representation of the shape is usually strongly nonlinear. Random searching algorithms, like genetic algorithms or simulated annealing approaches, are usually required to achieve globally optimal solution. However, most random-searching algorithms are slow and unreliable because of its sensitivity to some heuristicallyselected parameters. In this paper, we present a landmarksliding algorithm, which iteratively updates the currently estimated landmarks $\mathbf{V}$ to achieve a local optima. Together with the initialization method to be introduced in Section 3.1 , this landmark-sliding method is able to produce very accurate and reliable shape correspondence. Experiments in Section 3 also provide some evidence that this landmarksliding algorithm is in fact not very sensitive to the initial estimate of $\mathbf{V}$ (or $\mathbf{s}$ ).

The basic principle of the landmark sliding algorithm is to freely slide an initially-estimated $\mathbf{V}$ along the target shape $\mathbf{v}(s), 0 \leq s \leq L$ to minimize the cost function (7). We tackle the nonlinearity of the optimization problem by alternately repeating two steps: (a) sliding all estimated landmarks along the tangent directions, and (b) projecting the updated landmarks back to the target shape. Let $\mathbf{s}=\left\{s_{0}, s_{1}, \ldots, s_{n-1}\right\}$ be the parameters for the currently estimated landmarks $\mathbf{V}=\left\{\mathbf{v}_{0}, \mathbf{v}_{1}, \ldots, \mathbf{v}_{n-1}\right\}$. Our task is to seek an improved estimate of parameters $\mathbf{s}^{\prime}=\left\{s_{0}^{\prime}, s_{1}^{\prime}, \ldots, s_{n-1}^{\prime}\right\}$ or equally, estimate of landmarks $\mathbf{V}^{\prime}=\left\{\mathbf{v}_{0}^{\prime}, \mathbf{v}_{1}^{\prime}, \ldots, \mathbf{v}_{n-1}^{\prime}\right\}$.

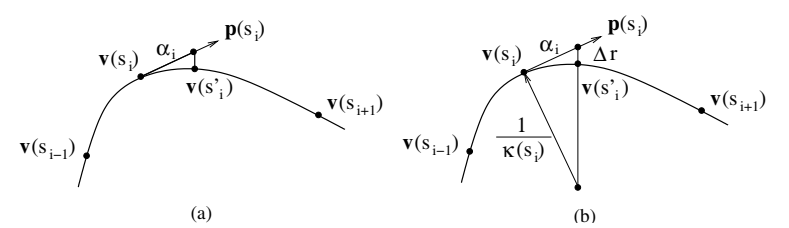

Figure 2: Illustrations of the landmark sliding and projection: (a) landmark sliding along tangent direction, followed by a projection back to the shape contour, and (b) selecting sliding step length based on the shape curvatures.

The normalized tangent direction at a landmark $\mathbf{v}(s)$ can be calculated by

$$
\mathbf{p}(s)=\left[\begin{array}{c}
p_{x}(s) \\
p_{y}(s)
\end{array}\right]=\left[\begin{array}{c}
\frac{\dot{x}(s)}{\sqrt{\dot{x}^{2}(s)+\dot{y}^{2}(s)}} \\
\frac{\dot{y}(s)}{\sqrt{\dot{x}^{2}(s)+\dot{y}^{2}(s)}}
\end{array}\right] .
$$

Let $\alpha_{i}$ be the sliding distance of $\mathbf{v}_{i}$ along tangent direction. Thus, $i$-th landmark after a step of sliding is at $\mathbf{v}_{i}+\alpha_{i} \mathbf{p}\left(s_{i}\right)$. After a step of sliding, those landmarks are usually not located along the original target shape any more. We address this problem by projecting them back to the target shape contour as

$$
\mathbf{v}_{i}^{\prime}=\mathbf{v}\left(s_{i}+\alpha_{i}\right)
$$

as illustrated in Fig. 2(a).

Considering this, shape correspondence through landmark sliding is to find an optimal $\boldsymbol{\alpha}=\left(\alpha_{0}, \alpha_{1}, \ldots, \alpha_{n-1}\right)^{T}$ minimizing the cost function

$$
\begin{aligned}
& \left(\mathbf{x}+\mathbf{P}_{x} \boldsymbol{\alpha}\right)^{T} \mathbf{L}\left(\mathbf{x}+\mathbf{P}_{x} \boldsymbol{\alpha}\right)+\left(\mathbf{y}+\mathbf{P}_{y} \boldsymbol{\alpha}\right)^{T} \mathbf{L}\left(\mathbf{y}+\mathbf{P}_{y} \boldsymbol{\alpha}\right) \\
& \left.+\lambda \sum_{i=0}^{n-1}\left\{\left(l_{i}+\alpha_{i}\right) \hat{l}_{i+1 \mid n}-\left(l_{i+1 \mid n}+\alpha_{i+1 \mid n}\right) \hat{l}_{i}\right)^{2}\right\},
\end{aligned}
$$

where matrices $\mathbf{P}_{x}=\operatorname{diag}\left(p_{x}\left(s_{0}\right), p_{x}\left(s_{1}\right), \ldots, p_{x}\left(s_{n-1}\right)\right)$ and $\mathbf{P}_{y}=\operatorname{diag}\left(p_{y}\left(s_{0}\right), p_{y}\left(s_{1}\right), \ldots, p_{y}\left(s_{n-1}\right)\right)$. We can calculate the updated parameters $\mathbf{s}^{\prime}$ by

$$
s_{i}^{\prime}=\left(s_{i}+\alpha_{i}\right) \mid L,
$$

and the updated curve-segment length $l_{i}$ by

$$
l_{i}^{\prime}=\left(s_{i+1}^{\prime}-s_{i}^{\prime}\right)\left|L=\left(l_{i}-\alpha_{i}+\alpha_{i+1}\right)\right| L .
$$

Finally, let's consider the topology-preservation constraint and other necessary additional constraints. Obviously, to preserve the shape topology, we only require

$$
l_{i}-\alpha_{i}+\alpha_{i+1}>0, i=0,1, \ldots, n-1,
$$

i.e., no landmark can move beyond its neighbors during the sliding/projection. 
Another problem is to constrain the error caused by the projection step, which may increase the shapecorrespondence cost. Considering the fact that the shapecorrespondence cost function is continuous with respect to the motion of landmarks, we control the possible landmarkcoordinate offsets from the projection step. As illustrated in Fig. 2(b), this can be controlled by limiting the allowed step-length $\alpha_{i}, i=0,1, \ldots, n-1$. It can be seen that, the larger the curvature at a landmark, the smaller the allowed step-length.

The curvature (without sign) at a landmark $\mathbf{v}(s)$ can be computed by

$$
\kappa(s)=\frac{|\dot{x}(s) \ddot{y}(s)-\ddot{x}(s) \dot{y}(s)|}{\left|\dot{x}(s)^{2}+\dot{y}(s)^{2}\right|^{\frac{3}{2}}} .
$$

As illustrated in Fig. 2(b), the osculating circle at a landmark should be of the radius $\frac{1}{\kappa}$. With an assumption of small step length, we take the approximation that a landmark after tangential sliding and the one after further projection are located along the same radius of the osculating circle. In this paper, we set the projection error to be less than $\Delta r$, it is easy to show that the step-length should satisfy the constraints

$$
\alpha_{i} \leq \sqrt{\frac{2 \Delta r}{\kappa\left(s_{i}\right)}+(\Delta r)^{2}}, i=0,1, \ldots, n-1 .
$$

Throughout this paper, we choose $\Delta r=0.5$ given that shapes are directly extracted from real images and the coordinates of landmarks are the corresponding row and column numbers in the original images.

It can be seen that minimizing the cost function (8) subject to constraints (9) and (10) is a simple quadratic programming problem, whose global optima can be efficiently calculated. For shape correspondence, we give an initial estimation of $\mathbf{s}$ or $\mathbf{V}$ and then repeatedly perform landmark sliding and projections until convergence. While no strict proof is provided to exclude possibilities of oscillation solutions, we do not see such a solution in all our experiments, with $\Delta r=0.5$. In the next section, we will discuss the selection of a good initial estimation of $\mathbf{V}$ and illustrate its performance by various examples.

\section{Experiment and Discussions}

In this section, we will first describe how to use the above algorithm for shape correspondence. Then we will present some experimental results. Finally, we will discuss some issues related to practical applications.

\subsection{Shape acquisition, landmark initializa- tion, and landmark sliding}

In order to use the proposed landmark-sliding algorithm for shape correspondence, we need parametric representations

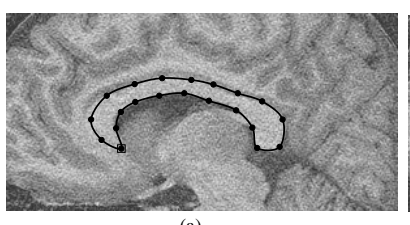

(a)
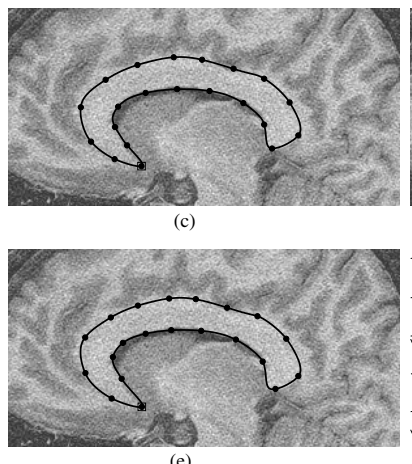
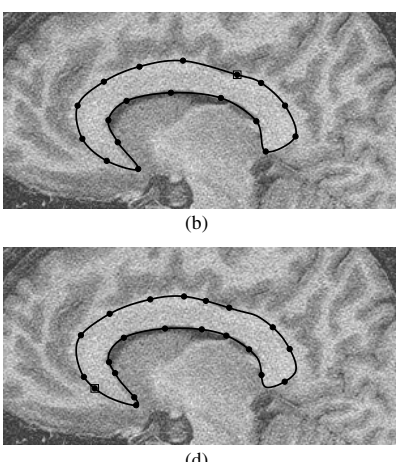

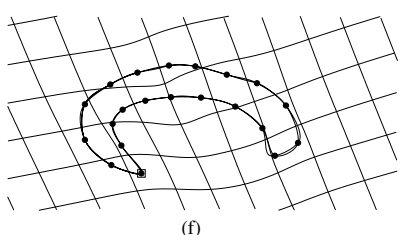

Figure 3: Illustrations of shape acquisition, landmark initialization and landmark sliding: (a) template shape constructed from a set of labelled landmarks, (b) target shape constructed from different number of labelled landmarks, (c) initial estimate $\mathbf{V}$ with $\lambda=+\infty$, (d) another initial estimate $\mathbf{V}$ with more bias to the desired locations, (e) corresponded landmarks. (f) the underlying deformation field for (e) (bending energy 0.2167 ). The curves in (f) are the target shape and deformed template shape calculated by Eq. (1), respectively.

of both the template and target shapes and an initial estimate of the target landmarks $\mathbf{V}$. In this section, we discuss these issues in a systematic way which aims for statistical shape analysis.

To build a statistical shape model of an object, each (training) shape sample is usually obtained, either manually or automatically, by labelling a set of landmarks in an image. In this paper, we adopt the Catmull-Rom splines to interpolate those labelled landmarks and construct the parametric representations of the shapes. The Catmull-Rom spline is used because of its smoothness and interpolation property, as illustrated in Figs. 3(a) and (b). We can use the labelled landmarks in the template shape as the template landmarks $\mathbf{U}$ for shape correspondence. However, it is usually not feasible to use the labelled landmarks in the target shape as an initial estimate of $\mathbf{V}$. The reasons are two-fold: (a) the labelled landmarks in the target may be of different number from those in the template; (b) the landmark labelling may start from any location along the target shape and therefore, the labelled landmarks in the target shape may not be a good initialization. An example is shown in Fig. 3(b), which contains different number of landmarks as those in (a). Also the relative location of the first landmark (shown in square) in the target shape is largely different from that in the template shape. Because 
the proposed landmark-sliding algorithm is a local search algorithm, big bias in the initial estimate may result in convergence to local optimum far from the desired correspondence.

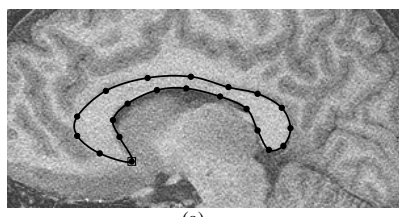

(a)
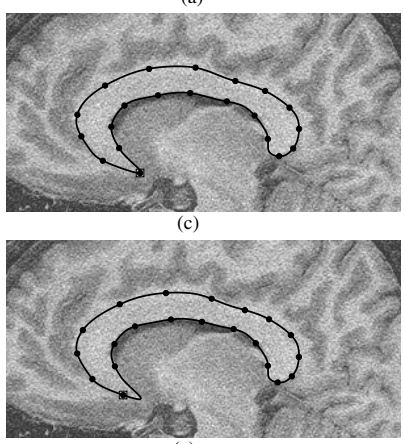

(e)

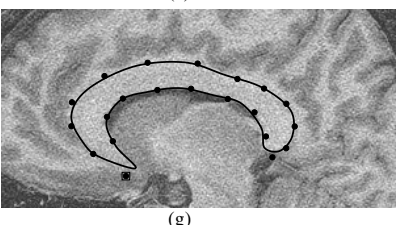

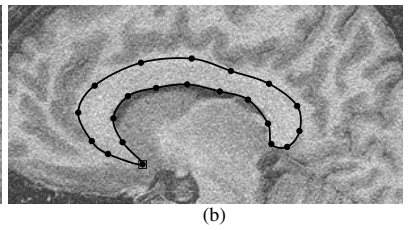
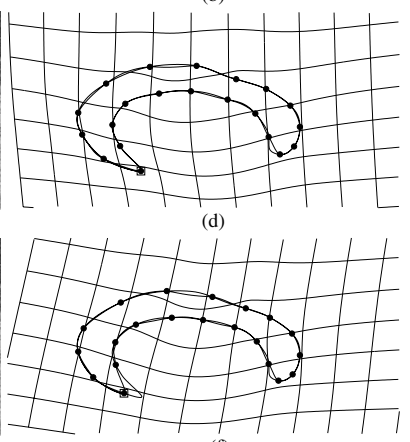

(f)

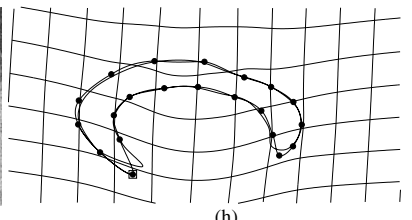

Figure 4: Callosum shape correspondence by landmark sliding: (a) template shape and landmarks, (b) target shape and landmarks, (c) resulting landmarks using the proposed method with $\lambda=10^{-3}$. (e) results using the proposed method with $\lambda=0$. (g) results using Bookstein's method. (d), (f), and (h) are deformation fields corresponding to (c), (e), and (g), with bending energy $0.2971,0.2442$ and 0.2394 , respectively. Target shapes are also shown in (c)(h) for elucidation.

There are many ways to achieve an initial rough correspondence, like those successfully used for shape recognition and retrieval, as reviewed in the section 1. In this section, we use a simple but effective algorithm to seek a good initial estimate of $\mathbf{V}$ based on the cost function (7). The basic principle is to set $\lambda=+\infty$ such that a strict equality instead of an approximate equality is held in (6). With this strict equality constraint, we can thoroughly try every possible point along the target shape as $\mathbf{v}_{0}$, which in fact uniquely defines a set of landmarks $\mathbf{V}$, because the curve length between each pair of landmarks in the target shape is fixed. Then we choose the one with smallest shape difference $d(\mathbf{U}, \mathbf{V})$ as the initial estimate of $\mathbf{V}$ for landmark sliding. In practice, we only need to check a finite set of possible $\mathbf{v}_{0}$ sampled along the target shape. This initial correspondence does not introduce any free parameters and any local optima. Meanwhile, it has a close relationship with the refined shape-correspondence method developed in Section 2. An example is illustrated in Fig. 3(c).

Figures 3(e) and (f) show the final corresponded landmarks in the target shape using the landmark-sliding algorithm and initial estimate shown in (c). Without special indication, we set $\lambda=10^{-3}$ in all our experiments. It can be seen that the landmark in the target and template are well corresponded (in terms of the thin-plate deformation) and the achieved landmarks also represent the target shape very accurately. In fact, the landmark sliding algorithm has a certain level of robustness to the initialization error. Figure 3(d) gives another initial landmark estimate where $\mathbf{v}_{0}$ is farther from the desired location and initial landmark distribution along the shape is quite different from the desired one. As expected, this initial estimate also converges to the same shape correspondence results as shown in Figs. 3(e) and (f), using the proposed landmark sliding algorithm.

Figure 4 demonstrates the comparison results of shape correspondence using various methods. Figures 4(c) and (e) show the shape correspondences using the proposed method with $\lambda=10^{-3}$ and $\lambda=0$, respectively. We can see that, without considering the representation error $R(\mathbf{s})$, e.g., $\lambda=0$, the obtained $\mathbf{V}$ has smaller shape difference with the template $\mathbf{U}$, but they do not represent the target shape very well, as witnessed by the curve segment to the right of the first landmark (with square). Figure 4(g) shows the correspondence result using Bookstein's method [3], where landmarks may be moved away from the underlying shape, as witnessed by the first landmark.

\subsection{Corresponding shapes with open-curve or multiple curves}

Although shapes consisting of a single closed curve are very common in practice, open-curve shapes and multiple-curve shapes are also very important in statistical shape modelling. An open-curve shape may appear when part of the object is occluded or outside of the view. A multiple-curve shape is very important when we are simultaneously interested in several structures in an object. While some previous methods [6] may have difficulties in dealing with those general cases, the proposed methods can be easily extended to address those general cases.

For shapes with open curves and multiple curves, the shape-difference term will stay unchanged in the cost function, because the thin-plate bending energy is not related to the connection order of landmarks for certain shapes. The differences lie in the representation term $R(\mathbf{s})$ and the additional constraints. From closed-curve shape to opencurve shape, we make the following changes: (a) the first landmark $\mathbf{v}_{0}$ and the last landmark $\mathbf{v}_{n-1}$ should be fixed in the landmark sliding. This brings us additional linear constraints $\alpha_{0}=0$ and $\alpha_{n-1}=0$. (b) In defining $R(\mathbf{s})$ and topology-preservation constraint, we remove the term corresponding to the curve segment between $\mathbf{v}_{0}$ and $\mathbf{v}_{n-1}$. Fig. 5 shows an example of using landmark-sliding algo- 

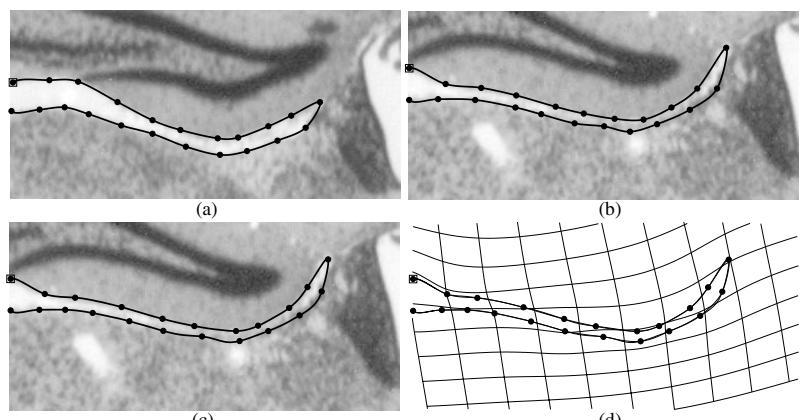

(d)
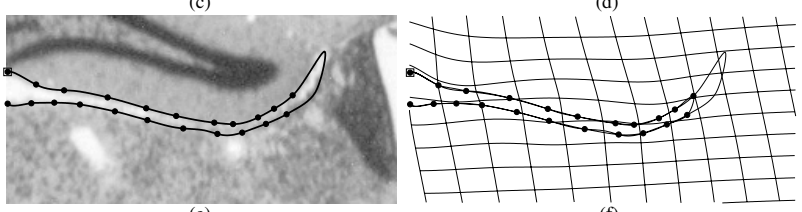

(f)
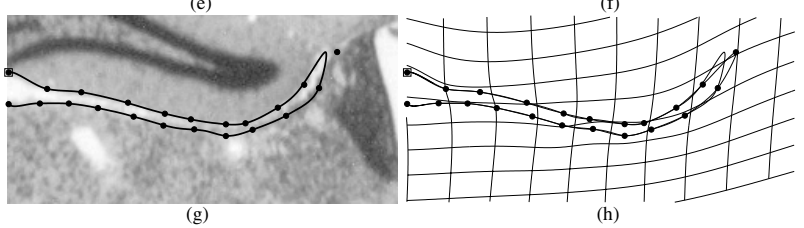

$(\mathrm{h})$

Figure 5: Stria-shape correspondence. (a)-(g) are the same as those in Fig. 4 except that the bending energy for (c), (e), and $(\mathrm{g})$ are $0.2128,0.1463$ and 0.2571 , respectively.

rithm for open-curve shape correspondence. Similarly, we see that the resulted landmarks from Bookstein's method and the proposed method without $R(\mathbf{s})$ may not well represent the target shape, as witnessed by the top-right peak landmark in Fig. 5(e) and (g).

For multiple-curve shapes, we only need to consider the shape representation error $R(\mathbf{s})$ and the additional constraints for each open/closed curve separately and then combine them together into the cost function (7). In Fig. 6, we show an example of corresponding two shapes with multiple structures in the human brain. We can see that the proposed method deals with all the structures in a global way and is able to delineate the boundaries in terms of their intra- and inter-shape information.

Shape with self-crossing (see Fig. 1(c)) appears where two objects share a part of the boundary. In this case, what we need to do is to find and fix those crossing points in the sliding, i.e., setting the corresponding $\alpha=0$. All the other processing is the same as in the case of multiple-curve shape.

\subsection{Multiple shape correspondence and statis- tical shape modelling}

So far, we discussed algorithms for identifying landmarks in a target shape to match the known landmarks in a template shape. In a typical problem, however, it is required to identify $n$ corresponding landmarks in a set of shape sam-

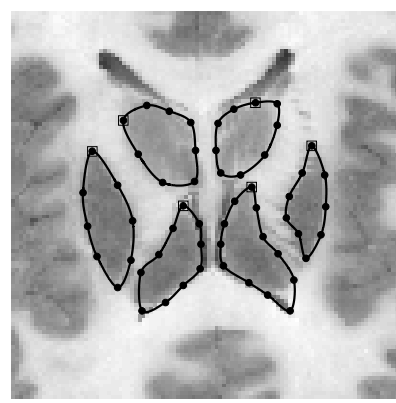

(a)

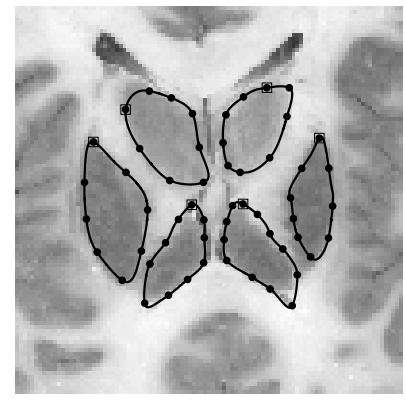

(c)

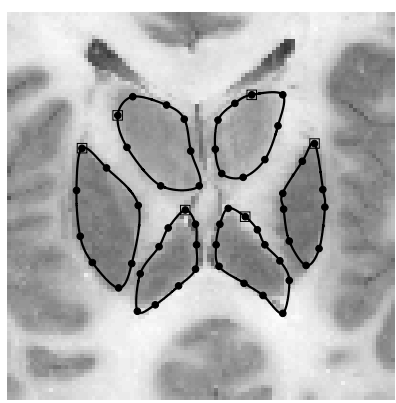

(b)

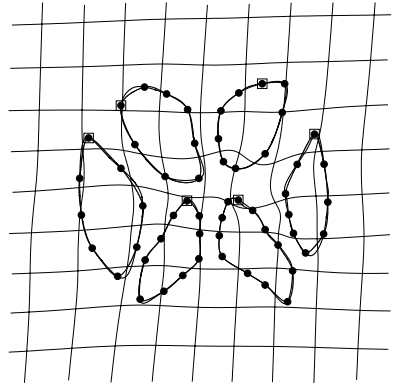

(d)
Figure 6: Shape correspondence of multiple structures in the brain: (a) template shape and landmarks, (b) target shape and initial landmarks, (c) corresponded landmarks, (d) the deformation field for (c), with bending energy 1.2238 .

ples. As discussed in [3], this can be accomplished by iteratively repeating the template-target shape correspondence algorithm. Basically, we first choose one shape sample as the template. Then we use the above landmark-sliding algorithm to correspond the landmarks in all the other shape samples. Based on this, we can compute a prototype shape by averaging all the shape samples with currently corresponded landmarks. All the shape samples are then recorresponded to this prototype shape and repeat the alternating shape averaging and correspondence algorithms until convergence.

As in [6], we use the statistical shape modelling to evaluate the performance of our algorithm. We extract 12 manually-corresponded callosum shapes from $12 \mathrm{MRI}$ brain slices (see supplementary material). Each shape consists of 24 landmarks, which are arranged in a 48dimensional vector containing $x$ - and $y$-coordinates of each landmark. Then we calculate the mean shape and covariance matrix for the given 12 shape vectors. The square roots of the eigenvalues of the covariance matrix represent the standard deviations $(\sigma)$ along the principal directions. In this experiment, the three largest deviations are [22.3031, 11.7680, 5.1857] before applying our multi-shape correspondence method and [16.3055, 8.9899, 4.3024] after applying 10 iterations of multiple-shape correspondence. It can be seen that the standard deviations in all three prin- 
cipal directions are decreased after shape correspondence. This shows that the shapes after correspondence generate a more compact statistical shape model.

Furthermore, we deform the mean (prototype) shapes, before and after 10 runs of multi-shape correspondence, by $3 \sigma$ or $-3 \sigma$ along each of these three principal directions. Figure 7 illustrates the deformed shapes with or without 10-runs of multi-shape correspondence. It can be seen that, through the shape correspondence, the statistical shape modelling is able to generate more reasonable shape samples. This is particularly evident in comparing the top-two images in the left-most column.

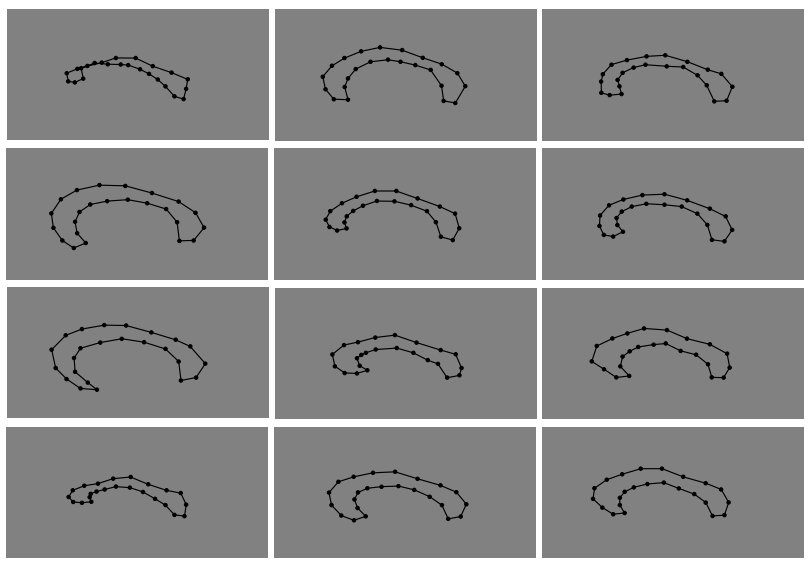

Figure 7: Multi-shape correspondence for statistical shape modelling. The first row is the deformed shapes before applying landmark-sliding algorithm. From left to right are the results by varying the first, second and third principal components by $-3 \sigma$, respectively. The second row is the deformed shapes after landmark-sliding-based shape correspondence. The third row and fourth row show the similar comparisons except that shapes are varied by $+3 \sigma$ in first three principal components.

\section{Conclusion}

This paper presented a novel landmark-based method for accurate shape correspondence. This method aligns multiple shape instances by identifying a set of corresponded landmark points along those shapes. The corresponded shapes can be used to generate more compact and accurate statistical shape models. Different from previous methods, we considered both global shape deformation and local geometric features in defining the shape-correspondence cost function to achieve a consistency between the landmark correspondence and the underlying shape correspondence. According to this cost function, we developed a novel landmark-sliding algorithm to achieve optimal shape correspondence. The proposed method is able to correspond a variety of $2 \mathrm{D}$ shapes, like closed-curve shapes, open-curve shapes, self-crossing shapes, and multiple-curve shapes. We also discuss the practical issue of landmark initializa- tion. The proposed method has been tested on various biological shapes in medical images and validated in constructing statistical shape models.

Acknowledgements-The authors would like to thank Anup Kedia for some help. This work was funded, in part, by NSF-EIA-0312861 and a grant from the University of South Carolina Research and Scholarship Fund.

\section{References}

[1] S. Belongie, J. Malik, and J. Puzicha. Shape matching and object recognition using shape contexts. IEEE Trans. Pattern Anal. Machine Intell., 24(24):509-522, April 2002.

[2] F. L. Bookstein. Principal warps: Thin-plate splines and the decomposition of deformations. IEEE Trans. Pattern Anal. Machine Intell., 11(6):567-585, June 1989.

[3] F. L. Bookstein. Landmark methods for forms without landmarks: Morphometrics of group differences in outline shape. Medical Image Analysis, 1(3):225-243, 1997.

[4] H. Chui and A. Rangarajan. A new algorithm for non-rigid point matching. In Proc. IEEE Conf. Computer Vision Pattern Recogn., pages 44-51, 2000.

[5] T. F. Cootes, C. J. Taylor, D. H. Cooper, and J. Graham. Active shape models - their training and application. Comput. Vision Image Understanding, 61(1):38-59, Jan. 1995.

[6] R. Davies, C. Twining, T. Cootes, J. Waterton, and C. Taylor. A minimum description length approach to statistical shape modeling. IEEE Trans. Med. Imag., 21(5):525-537, May 2002.

[7] Y. Gdalyahu and D. Weinshall. Flexible syntactic matching of curves and its application to automatic hierarchical classification of silhouettes. IEEE Trans. Pattern Anal. Machine Intell., 21(12):1312-1328, Dec. 1999.

[8] A. Hill and C. Taylor. A framework for automatic landmark identification using a new method of nonrigid correspondence. IEEE Trans. Pattern Anal. Machine Intell., 22(3):241-251, March 2000.

[9] D. G. Kendall, D. Barden, T. K. Carne, and H. Le. Shape and Shape Theory. John Wiley \& Sons, LTD, 1999.

[10] A. Kotcheff and C. Taylor. Automatic construction of eigenshape modles by direct optimization. Medical Image Analysis, 2:303-314, 1997.

[11] M. E. Leventon, E. L. Grimson, and O. Faugeras. Statistical shape influence in geodesic active contours. In Proc. IEEE Conf. Computer Vision Pattern Recogn., pages 316323,2000

[12] E. Petrakis, A. Diplaros, and E. Milios. Matching and retrieval of distorted and occluded shapes using dynamic programming. IEEE Trans. Pattern Anal. Machine Intell., 24(11):1501-1516, Nov. 2002.

[13] M. Powell. A thin plate spline method for mapping curves into curves in two dimensions. In Proc. Computational Techniques and Applications, pages 43-57, 1995.

[14] C. G. Small. The Statistical Theory of Shape. SpringerVerlag, 1996.

[15] Y. Wang, B. Peterson, and L. Staib. Shape-based 3d surface correspondence using geodesics and local geometry. In Proc. IEEE Conf. Computer Vision Pattern Recogn., pages (II)644-651, 2000. 